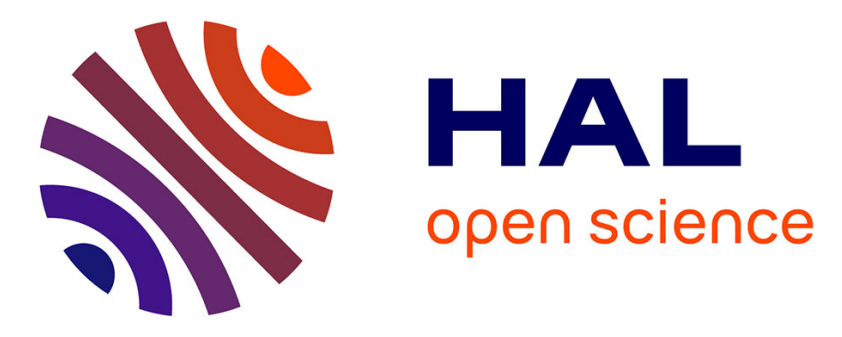

\title{
Dirichlet-to-Neumann semigroup acts as a magnifying glass
}

Mohamed Amine Cherif, Toufic El Arwadi, Hassan Emamirad, Jean-Marc

Sac-Epee

\section{- To cite this version:}

Mohamed Amine Cherif, Toufic El Arwadi, Hassan Emamirad, Jean-Marc Sac-Epee. Dirichletto-Neumann semigroup acts as a magnifying glass. Semigroup Forum, 2014, 88 (3), pp.753-767. 10.1007/s00233-014-9572-5 . hal-01095477

\section{HAL Id: hal-01095477 https://hal.science/hal-01095477}

Submitted on 15 Dec 2014

HAL is a multi-disciplinary open access archive for the deposit and dissemination of scientific research documents, whether they are published or not. The documents may come from teaching and research institutions in France or abroad, or from public or private research centers.
L'archive ouverte pluridisciplinaire HAL, est destinée au dépôt et à la diffusion de documents scientifiques de niveau recherche, publiés ou non, émanant des établissements d'enseignement et de recherche français ou étrangers, des laboratoires publics ou privés. 


\title{
DIRICHLET-TO-NEUMANN SEMIGROUP ACTS AS A MAGNIFYING GLASS
}

\author{
MOHAMED AMINE CHERIF, TOUFIC EL ARWADI, HASSAN EMAMIRAD \\ AND JEAN-MARC SAC-ÉPÉE
}

\begin{abstract}
The first aim of this paper is to illustrate numerically that the Dirichlet-to-Neumann semigroup represented by P. Lax acts as a magnifying glass. In this perspective, we used the finite element method for the discretization of the correspondent boundary dynamical system using the implicit and explicit Euler schemes. We prove by using the Chernoff's Theorem that the implicit and explicit Euler methods converge to the exact solution and we use the (P1)-finite elements to illustrate this convergence through a FreeFem ++ implementation which provides a movie available online. In the Dirichlet-to-Neumann semigroup represented by P. Lax the conductivity $\gamma$ is the identity matrix $I_{n}$, but for an other conductivity $\gamma$, the authors of [3] supplied an estimation of the operator norm of the difference between the Dirichlet-toNeumann operator $\Lambda_{\gamma}$ and $\Lambda_{1}$, when $\gamma=\beta I_{n}$ and $\beta=1$ near the boundary $\partial \Omega$ (see Lemma 2.1). We will use this result to estimate the accuracy between the correspondent Dirichlet-to-Neumann semigroup and the Lax semigroup, for $f \in H^{1 / 2}(\partial \Omega)$.
\end{abstract}

\section{INTRODUCTION.}

The semigroup character of a magnifying glass is intuitively quite obvious. From an optical point of view, the magnifying glass has to leave the viewing object unchanged if we look at it from a zero distance, while if we look at it from a distance $t$, and then from a distance $s$, it is as if we looked at it from a distance $t+s$. That is the semigroup character of a magnifying glass. Surprisingly, in his book [10, 36.2], P. Lax constructs an example of a $\left(C_{0}\right)$ semigroup who acts in the same way. He takes the Banach space $X=C\left(S^{n-1}\right)$, the space of continuous functions $f$ on the $n-1$ dimensional unit sphere or $X$ the Hilbert space $L^{2}\left(S^{n-1}\right)$. For each such function $f$ there is a uniquely determined harmonic function $u$,

Date: April 25, 2013.

1991 Mathematics Subject Classification. 35J25, 47F05, 47D06.

Key words and phrases. Dirichlet-to-Neumann operator, $\gamma$-harmonic lifting. 
called the harmonic lifting of $f$ in the $n$-dimensional unit ball $B$ which satisfies the following system:

$$
\begin{cases}\Delta u=0, & \text { in } B \\ u(\omega)=f(\omega), & \omega \text { in } S^{n-1}\end{cases}
$$

The Lax semigroup is defined by

$$
\mathrm{e}^{-t \Lambda_{1}} f(\omega)=u\left(e^{-t} \omega\right) \text { for } \omega \in S^{n-1} .
$$

In [10, 36.2 Theorem 1] it is shown that $\mathrm{e}^{-t \Lambda_{1}}$ forms a compact $\left(C_{0}\right)$ semigroup on $X$. Furthermore if $u$ is the harmonic lifting of $f$, then

$$
\lim _{t \rightarrow \infty}\left\|\mathrm{e}^{-t \Lambda_{1}} f-u(0)\right\|_{X}=0 .
$$

In fact for any $f \in X=C\left(S^{n-1}\right), 1.3$ is clear, since $u$ is uniformly continuous. For $f \in X=L^{2}\left(S^{n-1}\right)$, we can take for simplicity $n=2$ and the Fourier series expansion of $f$. The unique harmonic lifting of $f$ in $S^{n-1}$ can be expressed as

$$
u(r, \theta)=\frac{a_{0}}{2}+\sum_{k=1}^{\infty} r^{k}\left(a_{k} \cos k \theta+b_{k} \sin k \theta\right) .
$$

By replacing the Fourier coefficients by their integral expressions

$$
a_{k}=\frac{1}{\pi} \int_{0}^{2 \pi} f(\phi) \cos k \phi d \phi, \quad b_{k}=\frac{1}{\pi} \int_{0}^{2 \pi} f(\phi) \sin k \phi d \phi
$$

and using the identity

$$
1+2 \sum_{k=1}^{\infty} r^{k} \cos k(\theta-\phi)=\frac{1-r^{2}}{1-2 r \cos (\theta-\phi)+r^{2}}
$$

we get the Poisson integral expression of $u$

$$
u(r, \theta)=\frac{1}{2 \pi} \int_{0}^{2 \pi} \frac{1-r^{2}}{1-2 r \cos (\theta-\phi)+r^{2}} f(\phi) d \phi .
$$

Since $u(0)=\frac{1}{2 \pi} \int_{0}^{2 \pi} f(\phi) d \phi$, we get

$$
\int_{0}^{2 \pi}\left|u\left(e^{-t}, \theta\right)-u(0)\right|^{2} d \theta=\int_{0}^{2 \pi}\left|\frac{1}{2 \pi} \int_{0}^{2 \pi} \frac{2 e^{-t}\left(\cos (\theta-\phi)-e^{-t}\right)}{1-2 e^{-t} \cos (\theta-\phi)+e^{-2 t}} f(\phi) d \phi\right|^{2} d \theta .
$$

Now, for any $\varepsilon>0$ and any $(\theta, \phi) \in[0,2 \pi]^{2}$, we can take $t$ large enough such that $\left|\frac{2 e^{-t}\left(\cos (\theta-\phi)-e^{-t}\right)}{1-2 e^{-t} \cos (\theta-\phi)+e^{-2 t}}\right|<\varepsilon$. Consequently, by using the Cauchy-Schwarz inequality, 
one has

$$
\begin{aligned}
\left\|\mathrm{e}^{-t \Lambda_{1}} f-u(0)\right\|_{X}^{2} & =\int_{0}^{2 \pi}\left|u\left(e^{-t}, \theta\right)-u(0)\right|^{2} d \theta \\
& \leq \frac{\varepsilon}{2 \pi} \int_{0}^{2 \pi}\left(\int_{0}^{2 \pi}|f(\phi)| d \phi\right)^{2} d \theta \leq 2 \pi \varepsilon \int_{0}^{2 \pi}|f(\phi)|^{2} d \phi,
\end{aligned}
$$

which proves (1.3).

In each stage of the convergence (1.3), if we take $f_{t}(x)=u\left(e^{-t} x\right)$ as a function on the boundary, then according to the semigroup law $\mathrm{e}^{-s \Lambda_{1}} f_{t}(x)=u\left(e^{-(t+s)} x\right)$, this is just like taking a magnifying glass and going to the center of the disc. For visualization of this phenomena compare the Figures 1, 2 and 3, which present the Lax semigroup at time $t=0, t=0.5$ and $t=2$. At the end of the paper an internet address supplies the movie of this phenomena.

In [4], the authors showed that the Lax semigroup is in fact the Dirichlet-toNeumann (DtN) semigroup, when the domain $\Omega$ is the ball $B$ and the conductivity matrix, which will be defined later, is the identity matrix. To see this let us define the DtN semigroup in its whole generality as in [7]. We consider a bounded smooth domain $\Omega \subset \mathbb{R}^{n}$, and $\gamma$ an $n \times n$ symmetric matrix with smooth (for the simplicity of the exposition we take $\gamma \in C^{\infty}(\Omega)$ ) real elements in $\Omega$ which has uniformly bounded positive eigenvalues, i.e., there exists $0<c_{1}<c_{2}$ such that for every $x \in \Omega$ and $\xi \in \mathbb{R}^{n}$,

$$
c_{1}\|\xi\|^{2} \leq \xi^{T} \gamma(x) \xi \leq c_{2}\|\xi\|^{2} .
$$

This matrix is known as the electrical conductivity. Let $X:=L^{2}(\Omega)$ or $C(\bar{\Omega})$ and let the corresponding boundary space be $\partial X:=C(\partial \Omega)$ or $L^{2}(\partial \Omega)$. We solve the following Dirichlet problem

$$
\begin{cases}\nabla \cdot(\gamma \nabla u)=0, & \text { in } \Omega, \\ u=f, & \text { on } \partial \Omega .\end{cases}
$$

For any $f \in \partial X$, we write $u=L_{\gamma} f$. Such a function is called the $\gamma$-harmonic lifting of $f$ and $L_{\gamma}$ the $\gamma$-harmonic lifting operator. The function $u$ represents the electrical potential where this potential on the surface of the substance is $f$ and the substance is in electrical equilibrium. Now define the action of the generalized DtN operator on $f$ as the normal outward derivative of $u$ on the boundary, i.e.,

$$
\Lambda_{\gamma} f:=\left.(\nu \cdot \gamma \nabla u)\right|_{\partial \Omega} .
$$

In other words, we define $\Lambda_{\gamma}:=\nu(y) \cdot \gamma \nabla L_{\gamma}$ where $\nu(y)$ is the unit outer normal vector at $y \in \partial \Omega$, and $\Lambda_{\gamma}$ is called the DtN operator. In the simplest case where $\gamma$ is the identity matrix, we denote these operators by $L_{1}$, the harmonic lifting operator, and $\Lambda_{1}$, the corresponding DtN operator. 
We consider $\Lambda_{\gamma}$ as an unbounded operator on $\partial X$ with the domain,

$$
D\left(\Lambda_{\gamma}\right)=\left\{f \in \partial X: \Lambda_{\gamma} f \in \partial X\right\} .
$$

See $[1,6,12]$ for various properties of this domain. The operator $-\Lambda_{\gamma}$ generates an analytic compact semigroup of contractions on these spaces, $C(\partial \Omega)$ and $L^{2}(\partial \Omega)$. This semigroup can be identified as the trace of the solution to the following problem with dynamical boundary conditions:

$$
\begin{cases}\nabla \cdot(\gamma \nabla u(t, \cdot))=0, & \text { for every } t \in \mathbb{R}^{+}, \text {in } \Omega, \\ \partial_{t} u+\nu \cdot \gamma \nabla u=0, & \text { for every } t \in \mathbb{R}^{+}, \text {on } \partial \Omega, \\ u(0, \cdot)=f, & \text { on } \partial \Omega .\end{cases}
$$

In fact, by taking the trace of this solution (see [1] for uniqueness) and denoting it by $\left.u(t, x)\right|_{\partial \Omega}$, we can also define the DtN semigroup as,

$$
\mathrm{e}^{-t \Lambda_{\gamma}} f:=\left.u(t, x)\right|_{\partial \Omega}, \quad \text { for every } f \in \partial X
$$

It is easy to see that the $L^{2}(\partial \Omega)$ version of $\Lambda_{\gamma}$ is selfadjoint and nonpositive, and hence $\Lambda_{\gamma}$ generates also an analytic semigroup of maximal angle $\pi / 2$. Further properties such as contraction, compactness, positivity, irreducibility and Markov character of $\mathrm{e}^{-t \Lambda_{\gamma}}$ can be found in [12] and [4]. In [7], J. Escher showed that the operator $\Lambda_{\gamma}$ generates also in $C(\partial \Omega)$ an analytic semigroup of some positive angle $\theta$.

In the sequel, we are particularly interested in the case where $\gamma$ is the identity matrix $I_{n}$, so $u$ will satisfy the following system.

$$
\begin{cases}\Delta u(t, \cdot)=0, & \text { for every } t \in \mathbb{R}^{+}, \text {in } \Omega, \\ \partial_{t} u+\nu \cdot \nabla u=0, & \text { for every } t \in \mathbb{R}^{+}, \text {on } \partial \Omega, \\ u(0, \cdot)=f, & \text { on } \partial \Omega .\end{cases}
$$

In this case K.-J. Engel [6] showed that $\theta=\pi / 2$ even for the $C(\bar{\Omega})$ version of $\Lambda_{\gamma}$. This proves that for $\Omega=B$, we get (1.1) and in this case the DtN semigroup is the one given by (1.2). The main advantage of the Lax representation is that it is only necessary to solve the problem (1.1) in order to calculate the action of the semigroup in all times. In [5], it is shown that the Lax representation is optimal in the sense that for $\gamma=I_{n}$, the domain $\Omega$ should be a ball in $\mathbb{R}^{n}$.

The first aim of this paper is to illustrate numerically the action of the Lax semigroup and perhaps this permits to have an overview of the above open question. For achieving this, we used the finite element method for the discretization of (1.5). The program is performed by FreeFem ++ language (see [9]). This discretization is accomplished by using the implicit Euler scheme for the general case; i.e. $\gamma$ is not a constant multiple of the identity matrix but a function $\beta$ times the identity matrix. Thus, we have also to resolve the problem of accuracy. Since for $\beta=1$ in whole $B$ we have an explicit representation of the semigroup, 
it is convenient to consider a net $\beta_{\alpha}$ which converges to 1 as $\alpha \rightarrow 0$ and to check the numerical accuracy.

In [3], it is written that, although the following result may be considered wellknown, due to lack of a suitable reference we present a full proof for the reader's convenience. In this result the authors show that if the conductivity $\gamma=\beta I_{n}$ with $\beta=1$ near the boundary then one can estimate $\left\|\Lambda_{\gamma}-\Lambda_{1}\right\|_{\mathcal{L}\left(H^{1 / 2}(\partial \Omega), H^{s}(\partial \Omega)\right)}$ with respect to $\|\beta-1\|_{L^{\infty}(\Omega)}$. In the next section we cite again this result and we use it in order to compare $\Lambda_{1}$ with the general Dirichlet-to-Neumann operator with $\beta=1$ near the boundary; this estimation implies a strong estimation in $\partial X$ norm for the difference between $\mathrm{e}^{-t \Lambda_{\gamma}}$ and $\mathrm{e}^{-t \Lambda_{1}}$ which directly depends on $\|\beta-1\|_{L^{\infty}(\Omega)}$. As a consequence of this result, theoretically, the closer $\beta$ is to 1 , the closer $\mathrm{e}^{-t \Lambda_{\gamma}}$ is to $\mathrm{e}^{-t \Lambda_{1}}$. This shows the geometrical effects of the conductivity on the structure of the Dirichlet-to-Neumann semigroup.

The third section is divided into three parts. The first part is devoted to the Euler implicit scheme for our problem. To prove the convergence we construct an approximating family $\{V(t)\}_{t>0}$ and we use the Chernoff's product formula in order to theoretically prove the convergence in $L^{2}(\partial \Omega)$. Contrary to the explicit formulation (see [5, section 4]), here we do not have an explicit representation of $V(t)$. So we explicitly construct its left-inverse $W(t)$ and we define $V(t)$ as the right-inverse of $W(t)$. After proving the convergence, we use the finite element method via its variational formulation in order to numerically solve this problem. The second subsection is devoted to the explicit Euler scheme and we show why this method cannot be resolved by its variational formulation. Finally, in section 3.3, we consider an initial-boundary condition example which has a known harmonic lifting and we construct via Lax representation the solution at time $t=0.5$. Then we define a conductivity function $\gamma_{\alpha}=\beta_{\alpha} I_{n}$ such that $\beta_{\alpha} \rightarrow 1$, when $\alpha \rightarrow 0$ and we numerically illustrate that in this case $\mathrm{e}^{-t \Lambda_{\gamma}} \rightarrow \mathrm{e}^{-t \Lambda_{1}}$.

\section{COMPARISON OF THE SEMIGROUPS.}

In this section we show that if $\gamma=\beta I_{n}$ and $\beta=1$ in a neighborhood of $\partial \Omega$, then for $0 \leq t \leq T$ and any $f \in H^{1 / 2}(\partial \Omega)$ we have

$$
\left\|\mathrm{e}^{-t \Lambda_{\gamma}} f-\mathrm{e}^{-t \Lambda_{1}} f\right\|_{L^{2}(\partial \Omega)} \leq C_{T}\|\beta-1\|_{L^{\infty}(\Omega)},
$$

where the constant $C_{T}$ depends on $T, f$ and the constant $C$ in (2.1). The proof of this estimation is a straightforward consequence of the following lemma which has been proved in [3].

Lemma 2.1. Let $\beta \in C^{\infty}(\Omega)$ be a positive conductivity satisfying $\beta=1$ near $\partial \Omega$, then for all $s \in \mathbb{R}$, the following estimate holds

$$
\left\|\Lambda_{\gamma}-\Lambda_{1}\right\|_{\mathcal{L}\left(H^{1 / 2}(\partial \Omega), H^{s}(\partial \Omega)\right)} \leq C\|\beta-1\|_{L^{\infty}(\Omega)},
$$


where the constant $C$ depends on $s, \Omega$, the ellipticity constant of $\gamma$ and the support of $\beta-1$.

Now we are able to prove the main theorem of this section.

Theorem 2.1. For $\beta=1$ near $\partial \Omega$ such that $\beta \in C^{\infty}(\Omega)$ and $f \in H^{1 / 2}(\partial \Omega)$, there exists a constant $C_{T}$ depending on the ellipticity constant of $\gamma, f$ and $T$ such that

$$
\left\|\mathrm{e}^{-t \Lambda_{\gamma}} f-\mathrm{e}^{-t \Lambda_{1}} f\right\|_{L^{2}(\partial \Omega)} \leq C_{T}\|\beta-1\|_{L^{\infty}(\Omega)},
$$

for all $t \in[0, T]$.

Proof. One can readily write

$$
\begin{aligned}
\mathrm{e}^{-t \Lambda_{\gamma}} f-\mathrm{e}^{-t \Lambda_{1}} f & =\int_{0}^{t} \frac{d}{d s}\left(\mathrm{e}^{(s-t) \Lambda_{1}} \mathrm{e}^{-s \Lambda_{\gamma}} f\right) d s \\
& =\int_{0}^{t} \mathrm{e}^{(s-t) \Lambda_{1}}\left(\Lambda_{1}-\Lambda_{\gamma}\right) \mathrm{e}^{-s \Lambda_{\gamma}} f d s
\end{aligned}
$$

Since the semigroups $\mathrm{e}^{-t \Lambda_{\gamma}}$ and $\mathrm{e}^{-t \Lambda_{1}}$ are the analytic and contraction semigroups, then for any $f \in H^{1 / 2}(\partial \Omega)$ we have $\mathrm{e}^{-t \Lambda_{\gamma}} f \in H^{1 / 2}(\partial \Omega)$ and we get :

$$
\begin{aligned}
\left\|\mathrm{e}^{-t \Lambda_{\gamma}} f-\mathrm{e}^{-t \Lambda_{1}} f\right\|_{L^{2}(\partial \Omega)} & \leq \int_{0}^{t}\left\|\left(\Lambda_{1}-\Lambda_{\gamma}\right) \mathrm{e}^{-s \Lambda_{\gamma}} f\right\|_{L^{2}(\partial \Omega)} d s \\
& \leq C_{T}\|\beta-1\|_{L^{\infty}(\Omega)}
\end{aligned}
$$

where $C_{T}=C M_{T}$, with

$$
M_{T}=\sup _{t \in[0, T]} \int_{0}^{t}\left\|\mathrm{e}^{-s \Lambda_{\gamma}} f\right\|_{H^{1 / 2}(\partial \Omega)} d s .
$$

\section{Numerical Results}

Here we are going to approximate the Dirichlet-to-Neumann semigroup by means of Chernoff's Theorem. Let us recall this Theorem which is proved in [2].

Theorem 3.1 (Chernoff's product formula). Let $X$ be a Banach space and $\{V(t)\}_{t \geq 0}$ be a family of contractions on $X$ with $V(0)=I$. Suppose that the derivative $V^{\prime}(0) f$ exists for all $f$ in a set $\mathcal{D}$ and that the closure $\Lambda$ of $\left.V^{\prime}(0)\right|_{\mathcal{D}}$ generates a $\left(C_{0}\right)$ semigroup $S(t)$ of contractions. Then, for each $f \in X$,

$$
\lim _{n \rightarrow \infty} V\left(\frac{t}{n}\right)^{n} f=S(t) f
$$

uniformly for $t$ in compact subsets of $\mathbb{R}^{+}$. 
This procedure was achieved in [4] by choosing the approximating family $[V(t) f](x)=u\left(\mathrm{e}^{-t \gamma(x)} x\right)$, where $|x|=1$ and $\Omega=\left\{x \in \mathbb{R}^{n}:|x|<1\right\}$. In [5] the approximation family $\{V(t)\}_{t \geq 0}$ is chosen for the Euler explicit scheme, with

$$
V(t) f(x)= \begin{cases}(1-\alpha) u(x)+\alpha u\left(x-\alpha^{-1} t \gamma(x) \nu(x)\right), & 0 \leq t \leq \alpha T \\ V(\alpha T) f(x), & t>\alpha T\end{cases}
$$

where $u=L_{\gamma} f$ and $x \in \partial \Omega$. By using Theorem 3.1, it is proved that the following Euler explicit scheme

(EES)

$$
\begin{cases}\operatorname{div}\left(\gamma \nabla u^{m+1}\right)=0 & \text { in } \Omega \\ \frac{1}{\Delta t}\left(u^{m+1}-u^{m}\right)+\frac{\partial u^{m}}{\partial \nu_{\gamma}}=0 & \text { on } \partial \Omega \\ u^{0}=f & \text { on } \partial \Omega\end{cases}
$$

converges to the solution of the problem (1.5).

For the Euler implicit scheme one can provide a variational formulation programmable in finite element method of the problem.

For this purpose we replace the time derivative by

$$
\frac{\partial u}{\partial t}(m \Delta t, x) \simeq \frac{1}{\Delta t}\left(u^{m+1}(x)-u^{m}(x)\right), \quad \text { for } x \in \partial \Omega, \text { and } m=0, \cdots,[T / \Delta t],
$$

where $u^{m}(x)=u(m \Delta t, x)$. So, the Euler implicit scheme for the discrete boundary dynamical system can be written as

$$
\begin{cases}\operatorname{div}\left(\gamma \nabla u^{m+1}\right)=0 & \text { in } \Omega, \\ \frac{1}{\Delta t}\left(u^{m+1}-u^{m}\right)+\frac{\partial u^{m+1}}{\partial \nu_{\gamma}}=0 & \text { on } \partial \Omega \\ u^{0}=f & \text { on } \partial \Omega\end{cases}
$$

Since any $x$ with $|x|=1$ belongs to $\partial \Omega$, we have

$$
\frac{\partial u^{m+1}}{\partial \nu_{\gamma}} \approx \frac{u^{m+1}(x)-u^{m+1}(x-\Delta x \gamma(x) x)}{\Delta x} .
$$

By replacing (3.2) in (EIS), we get

$$
\left(1+\frac{\Delta t}{\Delta x}\right) u^{m+1}(x)-\frac{\Delta t}{\Delta x} u^{m+1}(x-\Delta x \gamma(x) x)=u^{m}(x) .
$$

Now let $0<\alpha \leq 1$ be a parameter. Since $\beta(x) \geq C^{-1}$ everywhere $x \in \partial \Omega$, there exists $T>0$ such that for all $t \leq T$, we have $x-t \gamma(x) \nu(x) \in \bar{\Omega}$. We may construct a family of operators in $\mathscr{L}\left(L^{2}(\partial \Omega)\right)$ as follows. Set

$$
W(t) f(x)= \begin{cases}(1+\alpha) u(x)-\alpha u\left(x-\alpha^{-1} t \gamma(x) \nu(x)\right), & 0 \leq t \leq \alpha T, \\ W(\alpha T) f(x), & t>\alpha T\end{cases}
$$


where $u$ is the $\gamma$-harmonic lifting of $f$. By taking $\alpha=\frac{\Delta t}{\Delta x}$ we can write (3.3) as

$$
W(\Delta t) u^{m+1}(x)=u^{m}(x)
$$

Now we define the family $V(t)$ as the inverse of $W(t)$ in the following sense

$$
W(t) V(t) f(x)=f(x)
$$

and we show that $V(t)$ satisfies all the assumptions of Chernoff's product formula in $X=L^{2}(\partial \Omega)$ with $\|\cdot\|=\|\cdot\|_{L^{2}(\partial \Omega)}$.

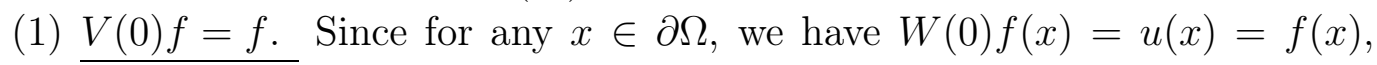
(3.6) shows

$$
f(x)=W(0) V(0) f(x)=V(0) f(x) .
$$

(2) $V^{\prime}(0) f=-\Lambda_{\gamma} f$. The derivative of $W(t)$ with respect to $t$ is

$$
W^{\prime}(t) f=\nabla u\left(x-\alpha^{-1} t \gamma(x) x\right) \gamma(x) x
$$

and at point $t=0$ we have $W^{\prime}(0) f=\Lambda_{\gamma} f$. Furthermore, the derivative of (3.6) gives $W^{\prime}(t) V(t)+W(t) V^{\prime}(t)=0$ and its evaluation at $t=0$, yields $\Lambda_{\gamma} f+V^{\prime}(0) f=0$

(3) $V(t)$ is of contraction. Let us first retrieve a lower bound for $W(t)$ in $L^{2}(\partial \Omega)$. In fact

$$
\|W(t) f\|^{2}=\int_{\partial \Omega}\left|u(x)+\alpha\left(u(x)-u\left(x-\alpha^{-1} t \gamma(x) x\right)\right)\right|^{2} d \sigma .
$$

Since $\left(u(x)-u\left(x-\alpha^{-1} t \gamma(x) x\right)\right) / \alpha^{-1}$ is an approximation of the normal derivative $\partial u / \partial \nu_{\gamma}$, so according to Hopf's Lemma (see [8]), this term is positive and consequently

$$
\int_{\partial \Omega}\left|u(x)+\alpha\left(u(x)-u\left(x-\alpha^{-1} t \gamma(x) x\right)\right)\right|^{2} d \sigma \geq \int_{\partial \Omega}|u(x)|^{2} d \sigma
$$

which implies that $\|W(t) f\| \geq\|f\|$. This implies that $\|f\|=\|W(t) V(t) f\| \geq$ $\|V(t) f\|$.

So we have proved that $V(t)$ satisfies all the assumptions of Chernoff's product formula and consequently the implicit Euler scheme converges to its exact solution.

The variational formulation of this problem can be obtained by multiplying both sides of the dynamic boundary condition by a test function $v$ and by using the divergence theorem, we get

$$
\int_{\Omega} \gamma \nabla u^{m+1} \nabla v d x-\int_{\partial \Omega} \gamma \frac{\partial u^{m+1}}{\partial n} v d \sigma=0
$$

that is

$$
\int_{\Omega} \Delta t \gamma \nabla u^{m+1} \nabla v d x+\int_{\partial \Omega} u^{m+1} v-\int_{\partial \Omega} u^{m} v d \sigma=0
$$


which is of the form

$$
a\left(u^{m+1}, v\right)=\ell(v)
$$

where

$$
a\left(u^{m+1}, v\right)=\int_{\Omega} \Delta t \gamma \nabla u^{m+1} \nabla v d x+\int_{\partial \Omega} u^{m+1} v d \sigma
$$

is the bilinear form with the unknown of the problem $u^{m+1}$ and

$$
\ell(v)=\int_{\partial \Omega} u^{m} v d \sigma
$$

is a linear form. This variational formulation can be resolved by finite elements implemented in FreeFem ++ and the result is illustrated in the following subsection.

3.1. Numerical illustration. We begin by taking the initial function $h(x, y)=$ $x^{2}+y$ on the boundary. The Dirichlet problem

$$
\begin{cases}\Delta u=0, & \text { in } \Omega \\ u=h, & \text { on } \partial \Omega\end{cases}
$$

admits a unique solution $u(x, y)=h(x, y)-g(x, y)$, where $g(x, y)=\left(x^{2}+y^{2}-\right.$ 1) $/ 2$. Since $g=0$ on the boundary of $\Omega, \Delta h=2$ and $\Delta g=2$. So the solution will be

$$
u(x, y)=\left(x^{2}-y^{2}\right) / 2+y+1 / 2 .
$$

The peculiarity of this function is that on the straight lines $y=-x+1$ for $(x, y) \in \mathbb{R}_{+} \times \mathbb{R}_{+}$and $y=x+1$ for $(x, y) \in \mathbb{R}_{-} \times \mathbb{R}_{+}$one has $u=1$ (see Figure 1).

Now we include the potential $\gamma \neq 1$. Define $\gamma_{\alpha}$ as

$$
\gamma_{\alpha}(x, y)= \begin{cases}1+\alpha \exp \left(\left(-\frac{9}{16}+x^{2}+y^{2}\right)^{-1}\right) & \text { if } x^{2}+y^{2}<\frac{9}{16} \\ 1 & \text { if } \quad x^{2}+y^{2} \geq \frac{9}{16}\end{cases}
$$

We remark that $1-\gamma_{\alpha}$ has its support in the disc $\mathcal{D}:=\{z \in \mathbb{C}:|z| \leq 3 / 4\}$, furthermore $\left\|1-\gamma_{\alpha}\right\|_{L^{\infty}(\Omega)}=\alpha \mathrm{e}^{-16 / 9}$, hence in order to justify our Theorem 2.1, we have to move $\alpha$ towards zero and observe the convergence of the solution to the exact solution given by (3.8). This is done in the subsequent Figures. Figure 4 to Figure 7 respectively illustrate the solution for $\alpha=300,50,10$ and 0.1 .

The calculations are performed via variational formulations described as above. We take 50 points on the circle $\partial \Omega$ and the domain inside is triangulated with Lagrange $P_{1}$ finite elements. The visualization is drawn indicating the isovalues of the finite element solution. We note the similarity of the exact solution (Figure 2) with the finite element solution for $\alpha=0.1$ (Figure 7). To illustrate this similarity we have computed at the end the difference between this solution and the exact solution in Figure 8. 


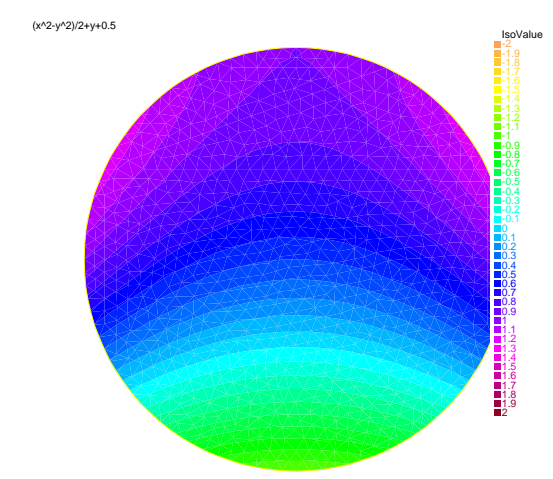

$$
\begin{gathered}
\text { Figure } 1 . \quad \text { Initial data } \\
u(0, x, y)=\left(x^{2}-y^{2}\right) / 2+y+1 / 2
\end{gathered}
$$

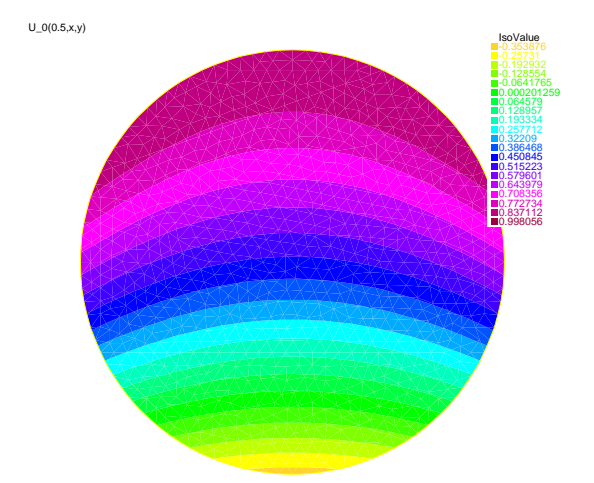

Figure 2. The exact solution at time $t=0.5$ $u(0.5, x, y)=\left((\exp (-0.5) x)^{2}-\left((\exp (-0.5) y)^{2}\right) / 2+\exp (-0.5) y+1 / 2\right.$.

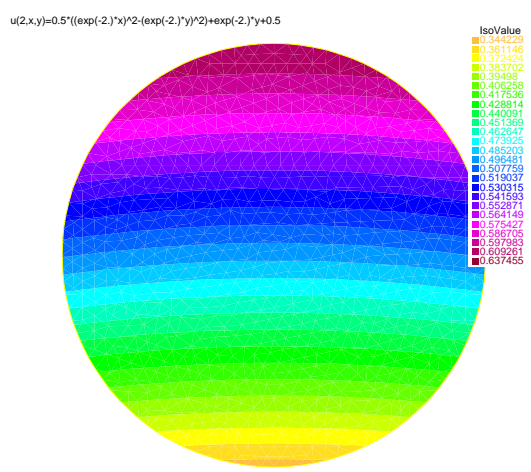

Figure 3. The exact solution at time $t=2$

$$
u(2, x, y)=\left((\exp (-2) x)^{2}-\left((\exp (-2) y)^{2}\right) / 2+\exp (-2) y+1 / 2\right. \text {. }
$$

Finally for illustrating the first part of this paper we have performed a movie with mp4 extension which is available online in

http://www.math.univ-metz.fr/ jmse/Emamirad 
Here we have taken the boundary function $f(x, y)=x^{4}+y^{2} \sin (2 \pi y)$, and 30 seconds after starting the movie res.mp4, we observe that we make a close-up of the center and that we approach the values of

$$
u(0)=\frac{1}{2 \pi} \int_{0}^{2 \pi}\left(\cos ^{4} \theta+\sin ^{2} \theta \sin (2 \pi \sin \theta)\right) d \theta=0.375
$$

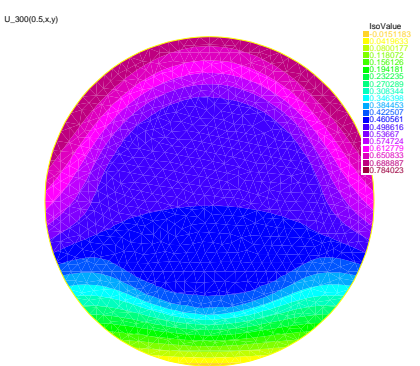

Figure 4. Solution for $\alpha=300$

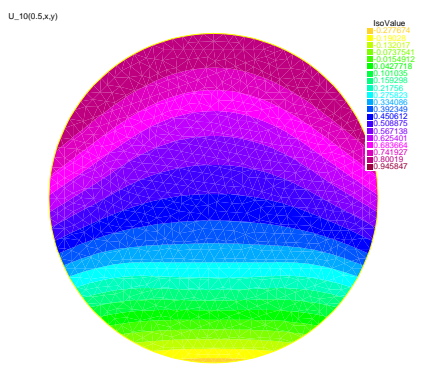

Figure 6. Solution for $\alpha=10$

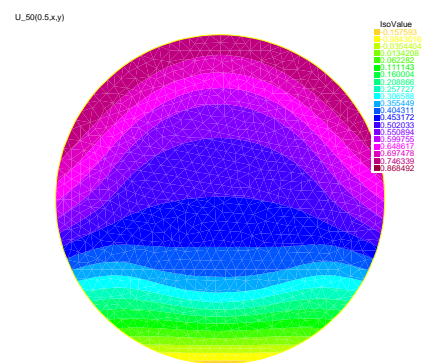

Figure 5. Solution for $\alpha=50$

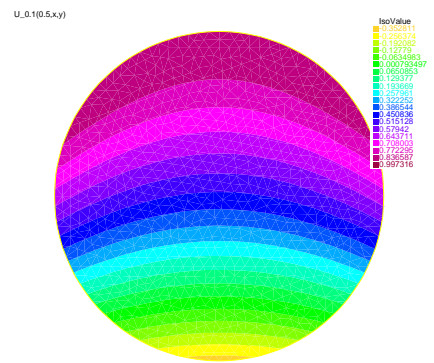

Figure 7. Solution for $\alpha=0.1$

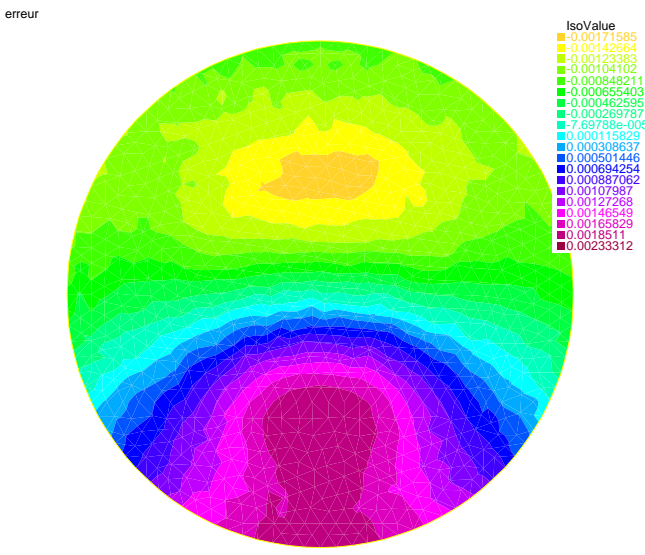

Figure 8. Error between $u_{e x a}$ and $u_{0.1}$ 


\section{REFERENCES}

[1] W. Arendt and A. F. M. Ter Elst, The Dirichlet-to-Neumann operator on rough domains. J. Differential Equations. 251 (2011), 2100-2124.

[2] P. R. Chernoff, Note on product formulas for operator semigroups. J. Funct. Analysis. 2 (1968), 238-242.

[3] H. Cornean, K. Knudsen, and S. Siltanen, Towards a D-bar reconstruction method for threedimensional eit. Journal of Inverse and Ill-posed Problems 12 (2006), $111-134$.

[4] H. Emamirad and I. Laadnani, An approximating family for the Dirichelet-toNeumann semigroup. Adv. Diff. Eqn. 11 (2006), 241-257.

[5] H. Emamirad and M. Sharifitabar, On Explicit Representation and Approximations of Dirichlet-to-Neumann Semigroup Semigroup Forum to appear .

[6] K.-J. ENGEL, The Laplacian on $C(\bar{\Omega})$ with generalized Wenzell boudary conditions., Arch. Math. 81 (2003), 548-558.

[7] J. Escher, The Dirichlet-Neumann operator on continuous functions. Annali della Scuola Norm. Sup. Pisa 21 (1994), 235-266.

[8] L. C. Evans, Partial Differential Equations Amer. Math. Soc., Providence, 1997.

[9] F. Hecht and O. Pironneau, A finite element software for PDE : FreeFem ++ , avaible online, http://www.freefem.org/ff ++ .

[10] P. D. Lax, Functional Analysis Wiley Inter-science, New-York, 2002.

[11] M. TAYlor, Partial Differential Equations II: Qualitative Studies of Linear Equations. Springer-Verlag, New-York. 1996.

[12] I. I. VRABIE, $C_{0}-$ Semigroups and Applications. North-Holland, Amsterdam, 2003.

†Departement de Mathématiques, Faculté des Sciences de Sfax, Université de Sfax, Route De Soukra Km 3.5, B.P.1171, 3000, Sfax, Tunisia

\#Department of Mathematics, Faculty of Science, Beirut Arab university, P.O. Box: 11-5020, BeIRUt,LEBANON

¥Laboratoire de Mathématiques, Université de Poitiers. teleport 2, BP 179, 86960 Chassneuil du Poitou, Cedex, France

$\dagger$ School of Mathematics, Institute for Research in Fundamental Sciences (IPM), P.O. Box 19395-5746, Tehran, Iran

\#\#aboratoire de Mathématiques et Applications de Metz, UMR 7122, Université de Lorraine - Metz, France

E-mail address: ${ }^{\dagger}$ mohamedamin.cherif@yahoo.fr

E-mail address: \#telarwadi@gmail.com

E-mail address: ${ }^{\ddagger}$ emamirad@math.univ-poitiers.fr

E-mail address: ${ }^{\dagger}$ emamirad@ipm.ir

E-mail address: ${ }^{\sharp}$ jean-marc.sac-epee@univ-lorraine.fr 